\title{
Carcinoma in the residual rectum of a long-standing Crohn's disease patient following subtotal colectomy: A case report
}

\author{
KAZUAKI SHIBUYA ${ }^{1}$, SHIGENORI HOMMA ${ }^{1}$, TADASHI YOSHIDA ${ }^{1}$, YOSUKE OHNO ${ }^{1}$, NOBUKI ICHIKAWA ${ }^{1}$, \\ HIDEKI KAWAMURA ${ }^{1}$, TEPPEI IMAMOTO ${ }^{2}$, YOSHIHIRO MATSUNO ${ }^{2}$ and AKINOBU TAKETOMI ${ }^{1}$ \\ ${ }^{1}$ Department of Gastroenterological Surgery I, Hokkaido University Graduate School of Medicine, Sapporo, Hokkaido 060-8638; \\ ${ }^{2}$ Department of Surgical Pathology, Hokkaido University Hospital, Sapporo, Hokkaido 060-8648, Japan
}

Received November 17, 2017; Accepted April 24, 2018

DOI: $10.3892 / \mathrm{mco} .2018 .1626$

\begin{abstract}
The development of colorectal cancer in long-standing Crohn's disease (CD) patients has become a major complication. Therapeutic guidelines for $\mathrm{CD}$-associated cancer (CDAC) have already been established in Western countries; however, specific guidelines are not currently available in Japan. Surveillance of the residual intestine for cancer screening is important for long-standing CD patients. The present case report describes the occurrence of rectal carcinoma in a patient with a 25-year history of CD. A 37-year-old male with a 17-year history of CD underwent semi-emergent subtotal colectomy and ileostomy for bowel obstruction secondary to the transverse colon stenosis, and multiple severe stenosis and inflammation. Postoperatively, the patient resumed pharmacological treatment and underwent follow-up colonoscopies at $~ 1-2$-year intervals. Despite continued pharmacological treatment, inflammation continued in the residual rectum. A total of 8 years following the primary operation, colonoscopy revealed inflammatory polyposis at the remnant rectum, which was diagnosed as adenocarcinoma. The interval between the last colonoscopy was 16 months. The patient then underwent laparoscopic abdominoperineal resection, and remained without recurrence for 12 months following resection. Thus, in long-standing CD patients, annual colonoscopy of the residual intestine may be considered for cancer screening, and specific surveillance guidelines for CDAC should be established.
\end{abstract}

Correspondence to: Dr Shigenori Homma, Department of Gastroenterological Surgery I, Hokkaido University Graduate School of Medicine, North 15, West 7, Kita-Ku, Sapporo, Hokkaido 060-8638, Japan

E-mail: homma.s@nifty.com

Abbreviations: APR, abdominoperineal resection; CA19-9, carbohydrate antigen 19-9; CD, Crohn's disease; CDAC, Crohn's disease associated cancer; CEA, carcinoembryonic antigen; CT, computed tomography; INF, infiltration; TNF, tumor necrosis factor

Key words: Crohn's disease, rectal cancer, residual intestine

\section{Introduction}

The development of colorectal cancer has become a major clinical concern among patients with long-standing Crohn's disease (CD) $(1,2)$, and research has shown there is a higher risk of colorectal cancer among CD patients $(3,4)$. With increased awareness of CD-associated cancer (CDAC), surveillance is deemed necessary, and specific guidelines for CDAC have already been established in Western countries $(5,6)$. In Japan, there is also guidelines for the therapy of inflammatory bowel disease but a surveillance for CDAC has not been decided. Unlike Western countries, anal canal cancer or carcinoma of the fistula is common in Japan as CDAC, which is prone to symptoms (2). As a result, the establishment of the surveillance for CDAC has been late but a national study is ongoing.

In $\mathrm{CD}$ patients, the principle of surgery is minimum resection and a prophylactic excision to avoid carcinogenesis cannot be selected. After all, surveillance for residual intestine for cancer screening is extremely important. We present a case of rectal carcinoma that occurred in a patient with a 25 -year history of CD.

\section{Case report}

A 37-year-old man with a 17-year history of CD visited the outpatient clinic at our hospital complaining of frequent diarrhea and weight loss. Colonoscopy showed severe stenosis and the formation of an internal fistula involving the stomach at the transverse colon, and multiple active inflammatory lesions with a longitudinal ulcer and stenosis involving the entire colon and rectum (Fig. 1A). Barium enema examination revealed shrinkage of the entire colon and stenosis at the transverse and sigmoidal colons (Fig. 1B). Computed tomography (CT) showed increased wall thickness across the entire colon and rectum. Two months after pharmacological treatment, bowel obstruction developed with dilatation of the small intestine (Fig. 2). An anal fistula and another evidence of carcinoma were not observed. We planned transverse colectomy with colostomy, but intraoperatively, the right-side colon showed extreme shrinkage and the ileocecal junction was hardly visible because it was involved in the transverse fistula. Despite severe inflammation in the rectum (Fig. 3A), we performed semi-emergent subtotal colectomy and ileostomy, and left 
the inflame rectum. Histopathological examination showed transmural inflammatory cell infiltrates with crypt abscess and granulomatous inflammation without any dysplasia or malignant lesions.

Postoperatively, the patient restarted medical therapy with infliximab, antibiotics, and azathioprine, and underwent surveillance colonoscopy at intervals of approximately 1-2 years for surveillance. Despite this pharmacological treatment, follow-up colonoscopy two years after surgery showed moderate inflammation in addition to erosion, stenosis, and an active ulcer in the residual rectum (Fig. 3B); however, dysplasia was not observed on biopsy. Active inflammation was observed once during remission four years after surgery (Fig. 3C), but relapsed to mild-to-moderate inflammation two years thereafter (Fig. 3D).

Eight years after the primary operation, he developed increased anal mucus discharge. Colonoscopy revealed inflammatory polyposis with erosion in the lower part of the remnant rectum (Fig. 4A), and biopsy of the polypoid lesions showed adenocarcinoma histologically (Fig. 5B). This was confirmed by immunohistochemical findings, consisting of diffuse strong nuclear staining of p53 (detected using BenchMark ULTRA, Roche, according to the recommendation by the manufacturer, including incubation with a Ready-to-Use antibody, clone DO-7, Roche, REF-No: 790-2912, diluted, for $16 \mathrm{~min}$, at $37^{\circ} \mathrm{C}$, incubated, for $64 \mathrm{~min}$, at $95^{\circ} \mathrm{C}$ ) (Fig. 5B), in addition to high $\mathrm{Ki}-67$ labeling index of $82.5 \%$ (detected using Bond-III, Leica, according to the recommendation by the manufacturer, including incubation with a monoclonal antibody, clone MIB-1, DAKO, REF-No: M7240, x200 diluted, for $15 \mathrm{~min}$, incubated, for $20 \mathrm{~min}$, at $100^{\circ} \mathrm{C}$ ) (Fig. 5B).

Laboratory examinations revealed a mild inflammatory reaction (white blood cell count: $5,500 / \mu 1$, neutrophil count: $3,867 / \mu 1$, C-reactive protein: $1.48 \mathrm{mg} / \mathrm{dl}$ ). The levels of the tumor markers, CEA and CA19-9, were $2.4 \mathrm{ng} / \mathrm{dl}$ and $11.8 \mathrm{U} / \mathrm{ml}$, respectively. CT and magnetic resonance imaging showed enhancement and thickening of the remnant rectal wall with no invasion into other organs or lymph nodes, and no distant metastasis (Fig 4B). He was diagnosed with rectal carcinoma and underwent laparoscopic abdominoperineal resection (APR). Using a perineal approach, an anal fistula at the right side of the rectum was resected. Histopathological examination revealed a well- to moderately-differentiated tubular adenocarcinoma, with a villotubular growth pattern (Fig. 6A and B) and with invasion into the subserosal tissue. The pathological classification was pT3N0, pStage IIA, according to UICC TNM Classification of MALIGNANT TUMOURS 8th for Cancer of the Colon and Rectum (7). In addition, chronic and active granulomatous inflammation was found in the surrounding rectum, consisting of atrophic distorted crypts with occasional crypt abscesses, transmural inflammatory cell infiltrate, fibrosis, and ill-defined epithelioid cell granulomas (Fig. 6C and D). These latter findings were histopathologically compatible with $\mathrm{CD}$ after treatment. Neither cytomegalovirus infection nor amyloid deposition was detected either histologically or immunohistochemically. The patient's postoperative course was uneventful, and he was discharged on postoperative day 33. He has been without recurrence for 12 months after APR.

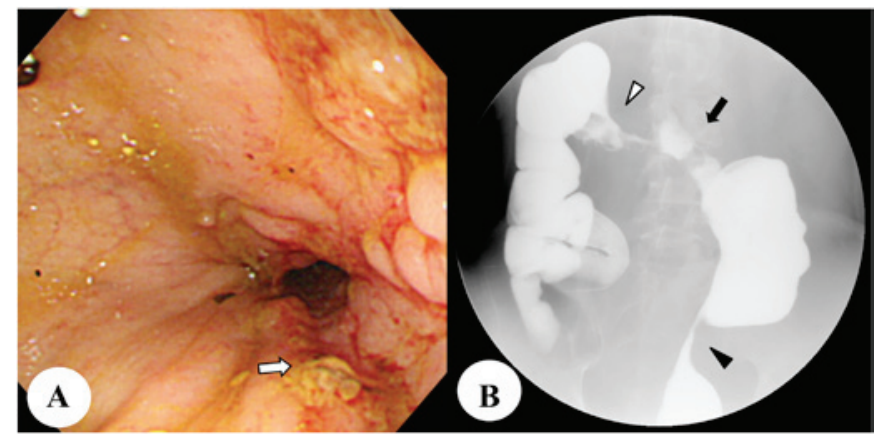

Figure 1. (A) Colonoscopy images showing the active lesions with a longitudinal ulcer (white arrow) at the transverse colon. (B) Barium enema examination revealed shrinkage of the entire colon and stenosis at the transverse (arrow) and sigmoidal colons (black arrow head). The white arrow head indicates the ileocecal junction.

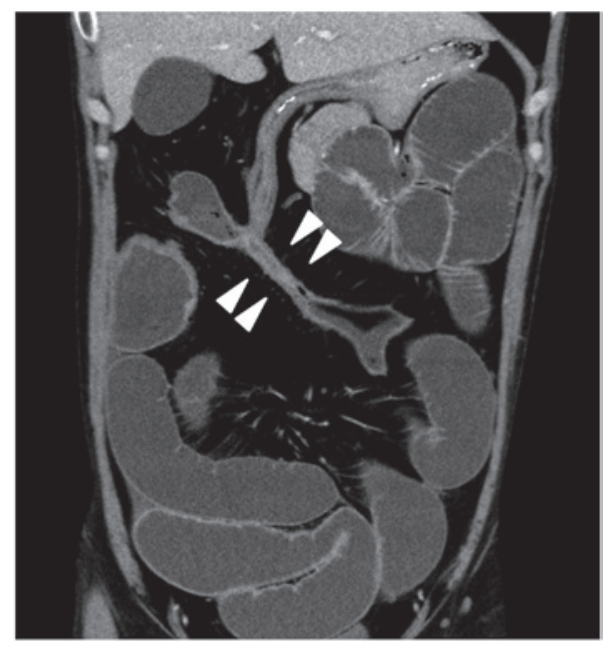

Figure 2. Abdominal computed tomographic image showing the increased wall thickness and stenosis in the transverse colon (white arrow heads) causing small bowel obstruction.

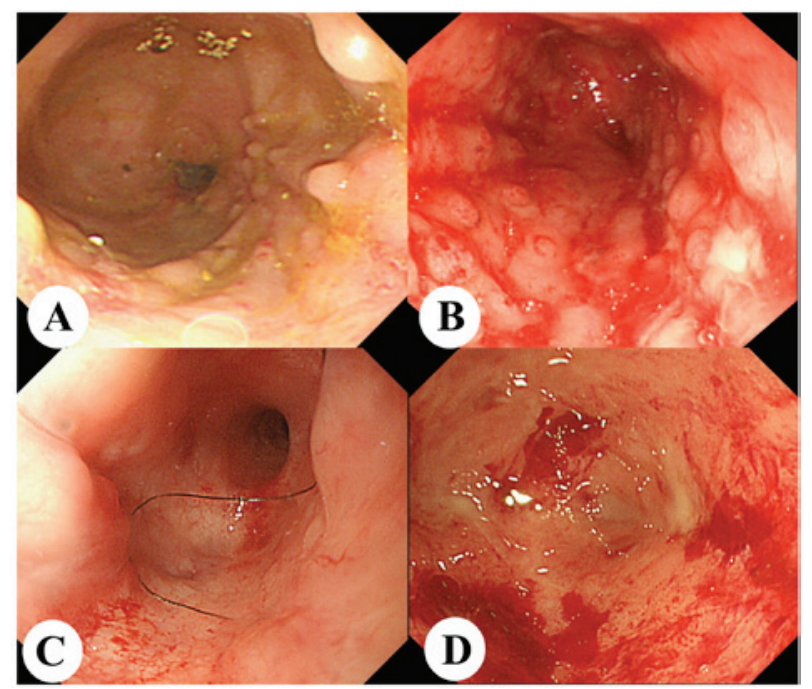

Figure 3. Pre- and postoperative colonoscopy in the rectum. (A) Preoperative colonoscopy showing severe inflammation, including an active ulcer, a scar from a longitudinal ulcer and stenosis. (B) Moderate inflammation in addition to erosion, stenosis and active ulcer were observed 2 years following surgery. (C) Active inflammation was not observed 4 years following surgery. (D) Mild-to-moderate inflammation including mucous redness and geographic ulcer were observed 6 years following surgery. 


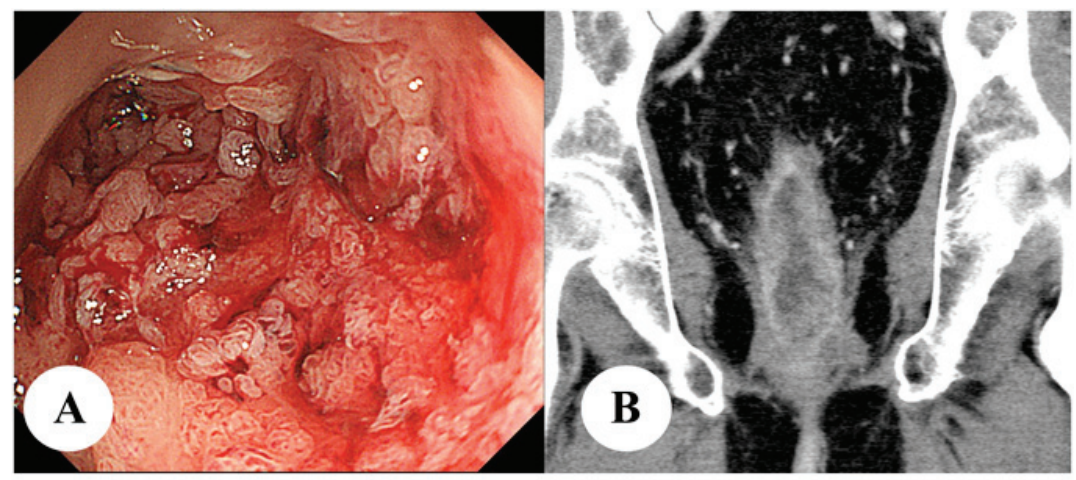

Figure 4. (A) Examinations were performed prior to the second operation: Colonoscopy revealed diffuse erosions with bleeding and 0-IIa polyposis, which were suspected to be dysplasia in the residual rectum. (B) Computed tomographic image showing the enhancement and wall thickening in the rectum.
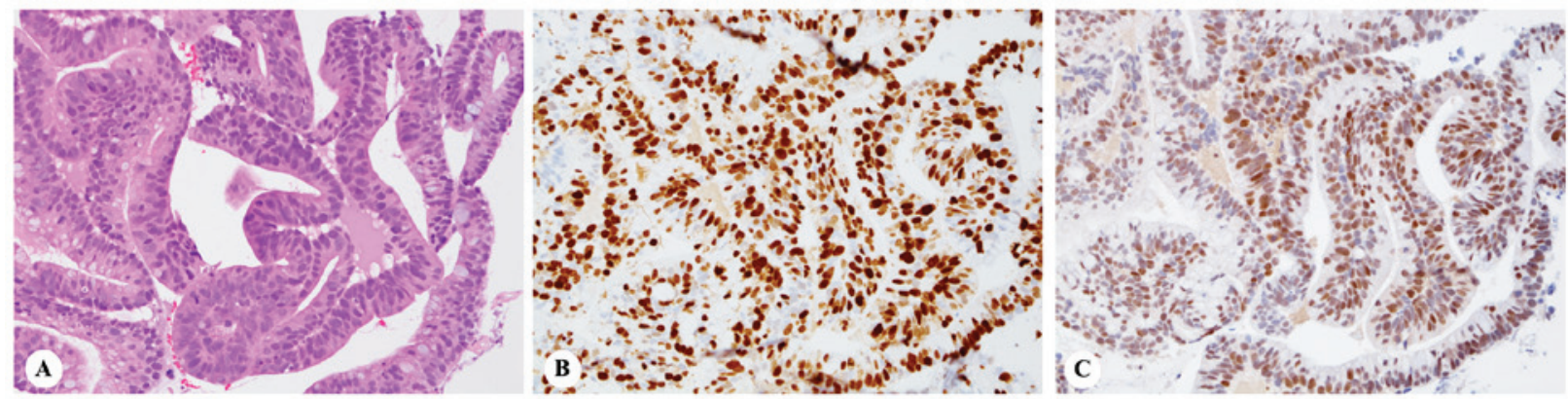

Figure 5. (A) Hematoxylin and eosin staining of the biopsy from the rectum. (B) Immunohistochemical detection of p53 and (C) immunohistochemical detection of Ki-67 (magnification, x200).

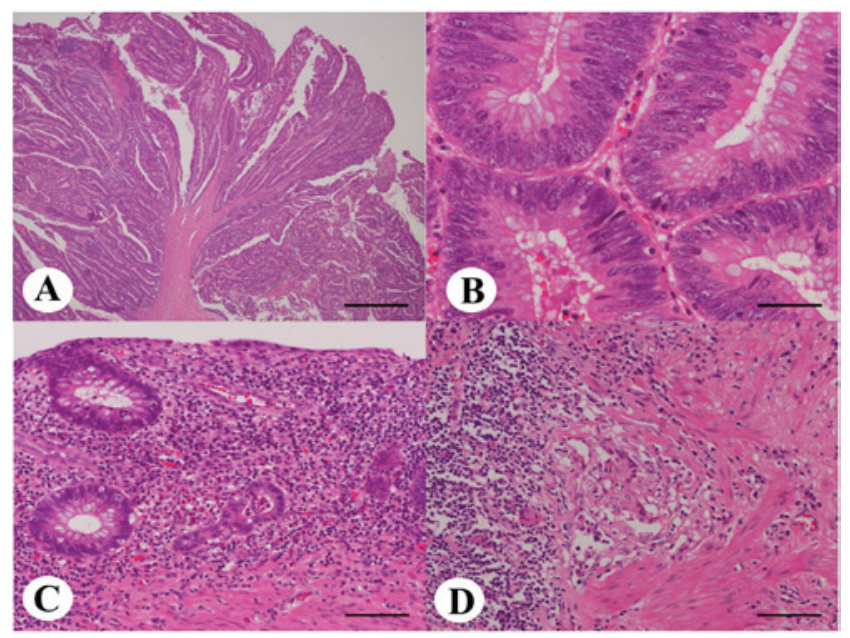

Figure 6. Histopathological examination using hematoxylin and eosin staining. (A) Low-power photomicrograph of the rectal adenocarcinoma showing the villotubular growth pattern (magnification, $\mathrm{x} 2$; scale bar, $1,000 \mu \mathrm{m}$ ). (B) High-power photomicrograph of the tubular adenocarcinoma (magnification, $\mathrm{x} 40$; scale bar, $50 \mu \mathrm{m}$ ). (C) Rectal mucosa neighboring the carcinoma; photomicrograph showing mixed inflammatory cell infiltrate and crypt abscess (magnification, x20; scale bar, $100 \mu \mathrm{m}$ ). (D) Photomicrograph showing the ill-defined epithelioid cell granuloma in the rectal proper muscular layer (magnification, x20; scale bar, $100 \mu \mathrm{m}$ ).

\section{Discussion}

The development of colorectal cancer has become a major complication among long-standing CD patients $(1,2)$. The first report describing CDAC was published in 1948 by
Warren et al (8), and several reports discussing the epidemiology of CDAC have followed. The risk of colorectal cancer in CD patients is 2.5 times that of the general population (3), and the mean duration of $\mathrm{CD}$ until diagnosis of colorectal cancer is 18.3 years (4). A dysplasia to carcinoma sequence has been associated with carcinoma in $\mathrm{CD}(5)$, and the risk of colorectal cancer has been reported to gradually increases according to disease duration in a recent meta-analysis, with an incidence of $0.4 / 1,000$ person years duration (pyd) in the $<10$ years' duration group, $0.8 / 1,000$ pyd in the $10-20$ years' duration group, and 1.2/1,000 pyd in the $>20$ years' duration group (4). More specifically in rectal cancer, the standardized relative risk has been reported to be 1.6 (9). In our patient, he was a high-risk patient because the disease duration was 25 years and the inflammation in the residual rectum had been continued. Because the principle of surgery in $\mathrm{CD}$ is minimum resection, however, APR as a prophylactic excision to avoid carcinogenesis could not be selected at the primary surgery. Therefore, appropriate repeat colonoscopy for residual intestine for cancer screening would be needed.

With awareness of CDAC, specific guidelines for CDAC was established in Western countries $(5,6)$, but it is undeveloped in Japan. According to Western guidelines, repeat colonoscopy over a 1- or 2-year interval is recommended for patients who have moderate-to-severe inflammation, and more frequent colonoscopy or colectomy is necessary for patients with dysplasia (5). This surveillance provides better prognosis in patients with inflammatory bowel disease, although it is difficult for colonoscopy alone to prevent carcinogenesis. Thus, the aim of surveillance is not to prevent the onset of 
carcinoma but to identify dysplasia or carcinoma at an early stage $(10,11)$. However, the location of carcinogenesis in CD is different from Western countries and Japan. The majority of cancer location is small bowel in Western countries (3), whereas anorectal carcinoma including cancer in anal fistulas counts 55\% of CDAC in Japan (2). Therefore, European surveillance protocol or guideline may not suitable for Japan wherein anorectal observation is more important. Our patient showed no evidence of dysplasia and therefore underwent colonoscopy, albeit irregularly, approximately every 1-2 years after surgery. The colonoscopy before the diagnosis of advanced carcinoma showed a persistent moderate inflammation that had persisted for over 20 years, and the interval was 16-month. Considering this history, our patient, who had a persistent inflammation of the residual intestine, should have undergone annual colonoscopy for cancer screening despite the absence of dysplasia. Thus, we recommend that CD patients with persistent inflammation in the residual intestine should be subjected to routine annual colonoscopy for cancer screening after surgery.

One more important point is that the resection of residual continuous inflammatory rectum should have been considered in his follow-up as a prophylactic resection. In ulcerative colitis, total proctocolectomy is generally performed to avoid carcinogenesis and continuous inflammation (12) but there has been no evidence that a prophylactic resection avoid carcinogenesis and improve prognosis of CD patients. To resect the residual rectum, APR was needed which required another surgical invasiveness and permanent artificial anus. Moreover, the inflammation could be expected to be improved with medical therapies and the lack of absolute surgical indication also decreased the necessity of APR as the next surgery.

In long-standing $\mathrm{CD}$ patients, annual colonoscopy for residual intestine may be considered for cancer screening, and specific surveillance guidelines should be established.

\section{Acknowledgements}

Not applicable.

\section{Funding}

No funding was received.

\section{Availability of data and materials}

All data generated or analyzed during this study are included in this published article.

\section{Authors' contributions}

$\mathrm{KS}, \mathrm{SH}, \mathrm{TY}, \mathrm{YO}, \mathrm{NI}$ and HK participated in the care of the patients. KS, SH, TY, YO, NI, HK, TI, YM and AT were all involved in collecting the patients' data. KS, TY, SH and AT considered the present cases based on the past literature and drafted the manuscript. KS was a major contributor to analyzing and interpreting the patient data, and to writing the manuscript. YM and TI performed the histological examination and data analysis. SH, TY and AT revised the manuscript. AT participated in critical revision of the manuscript. All authors read and approved the final manuscript.

\section{Ethics approval and consent to participate}

Written informed consent was obtained from the patient, and patient's anonymity was preserved.

\section{Consent for publication}

Written informed consent was obtained from the patient for the publication of any data and accompanying images.

\section{Competing interests}

The authors declare that they have no competing interests.

\section{References}

1. Jess T, Gamborg M, Matzen P, Munkholm P and Sørensen TI: Increased risk of intestinal cancer in Crohn's disease: A meta-analysis of population-based cohort studies. Am J Gastroenterol 100: 2724-2729, 2005.

2. Shinozaki M: Crohn's disease and intestinal cancer in Japan. J Jpn Soc Coloproctol 61: 353-363, 2008.

3. Canavan C, Abrams KR and Mayberry J: Meta-analysis: Colorectal and small bowel cancer risk in patients with Crohn's disease. Aliment Pharmacol Ther 23: 1097-1104, 2006.

4. Laukoetter MG, Mennigen R, Hannig CM, Osada N, Rijcken E, Vowinkel T, Krieglstein CF, Senninger N, Anthoni C and Bruewer M: Intestinal cancer risk in Crohn's disease: A meta-analysis. J Gastrointest Surg 15: 576-583, 2011.

5. Beaugerie L and Itzkowitz SH: Cancers complicating inflammatory bowel disease. N Engl J Med 372: 1441-1452, 2015.

6. Strong S, Steele SR, Boutrous M, Bordineau L, Chun J, Stewart DB, Vogel J and Rafferty JF; Clinical Practice Guidelines Committee of the American Society of Colon and Rectal Surgeons: Clinical Practice Guideline for the Surgical Management of Crohn's Disease. Dis Colon Rectum 58: 1021-1036, 2015.

7. National Comprehensive Cancer Network: NCCN Guidelines: Colon Cancer. Version 1.2017. https://www.ncen.org/patients/ guidelines/colon/files/assets/common/downloads/files/colon.pdf. Accessed January 9, 2018

8. Warren S and Sommers SC: Cicatrizing enteritis as a pathologic entity; analysis of 120 cases. Am J Pathol 24: 475-501, 1948.

9. Ekbom A, Helmick C, Zack M and Adami HO: Increased risk of large-bowel cancer in Crohn's disease with colonic involvement. Lancet 336: 357-359, 1990.

10. Friedman S, Rubin PH, Bodian C, Harpaz N and Present DH: Screening and surveillance colonoscopy in chronic Crohn's colitis: Results of a surveillance program spanning 25 years. Clin Gastroenterol Hepatol 6: 993-998; quiz 953-954, 2008.

11. Krugliak Cleveland N, Colman RJ, Rodriquez D, Hirsch A Cohen RD, Hanauer SB, Hart J and Rubin DT: Surveillance of IBD using high definition colonoscopes does not miss adenocarcinoma in patients with low-grade dysplasia. Inflamm Bowel Dis 22: 631-637, 2016.

12. Riddell RH, Goldman H, Ransohoff DF, Appelman HD, Fenoglio CM, Haggitt RC, Ahren C, Correa P, Hamilton SR, Morson BC, et al: Dysplasia in inflammatory bowel disease: Standardized classification with provisional clinical applications. Hum Pathol 14: 931-968, 1983. 